\title{
The Experimental Researches of Electrical Characteristics of the Gaas MIC Low-Noise Amplifier and the Switch-Off of Own Production for Equipment of Autonomous Spacecraft Radionavigation
}

\author{
Vadim N. Shkolniy ${ }^{a *}$, Sergey B. Suntsov ${ }^{\mathrm{a}}$, \\ Aleksey V. Kondratenko ${ }^{\text {b }}$, Dmitriy A. Shishkin ${ }^{\text {b, }}$ \\ Vladimir D. Dmitriev ${ }^{\mathrm{b}}$ and Vadim M. Karaban ${ }^{\mathrm{b}}$ \\ ${ }^{a}$ Academician M.F. Reshetnev Information Satellite Systems \\ 52 Lenin Str., Zheleznogorsk, Krasnoyarsk region, 662972, Russia \\ ${ }^{b}$ TUSUR \\ 40 Lenina Str, Tomsk, 634050, Russia
}

Received 20.02.2015, received in revised form 04.03.2015, accepted 27.03.2015

The presents of electrical characteristics experimental researches results of the radiation-hardened on-off switch and low-noise amplifier (LNA) monolithic integrated circuits (MIC) of the designed and manufactured based on arsenide-gallium technology of own production for use in the multi-channel on-board navigation receivers of global navigation satellite systems (GNSS) signals for autonomous coordinate and time support of all types orbits spacecraft.

Keywords: semiconductors, nanoheterostructures, arsenide-gallium technology, monolithic integrated circuits, spacecraft, autonomous navigation, on-off switch, low noise amplifier, experimental researches, electrical characteristics.

(C) Siberian Federal University. All rights reserved

* Corresponding author E-mail address: shkolniy@iss-reshetnev.ru 


\title{
Экспериментальные исследования
}

\section{электрических характеристик арсенид-галлиевых МИС малошумящего усилителя и двухпозиционного коммутатора собственного производства для аппаратуры автономной радионавигации космических аппаратов}

\author{
В.Н. Школьный ${ }^{a}$, С.Б. Сунцов ${ }^{\mathrm{a}}$, А.В. Кондратенко \\ Д.А. Шишкин ${ }^{0}$, В.Д. Дмитриев ${ }^{0}$, В.М. Карабан \\ a $\mathrm{AO}$ «Информачионные спутниковые системыл» \\ имени академика М.Ф. Решетнёва» \\ Россия, 662972, Красноярский край, Железногорск, ул. Ленина, 52 \\ ${ }^{\sigma}$ Томский государственный университет \\ систем управления и радиоэлектроники \\ Россия, 634050, Томск, пр. Ленина, 40
}

\begin{abstract}
Представлень результаты экспериментальных исследований электрических характеристик радиационностойких монолитно-интегральных схем двухпозиционного коммутатора и малошумящего усилителя, разработанного и изготовленного на основе арсенид-галлиевой технологии собственного производства для применения в составе бортовых многоканальных навигационных приёмников сигналов глобальных навигационных спутниковых систем космических аппаратов всех типов орбит для их автономного координатно-временного обеспечения.
\end{abstract}

Ключевые слова: полупроводники, наногетероструктуры, арсенид-галлиевая технология, монолитные интегральные схемь, космический аппарат, автономная навигация, двухпозиционный коммутатор, малошумящий усилитель, экспериментальнье исследования, электрические характеристики.

\section{Введение}

Координатно-временное обеспечение является одним из важнейших условий успешного функционирования космических аппаратов различного назначения, в особенности тех, полезная нагрузка которых представляет собой измерительные системы: активные и пассивные радиолокаторы, средства наблюдения и дистанционного зондирования, навигационные устройства и др. Особое значение координатно-временное обеспечение имеет в случаях совместного функционирования нескольких космических аппаратов, которые решают общую задачу.

Для функционирования на разных типах орбит (низких, геостационарных, высокоэллиптических) необходимо использовать несколько антенн (антенно-фидерных систем), которые посредством коммутатора подключаются к приёмнику сигналов глобальных навигационных спутниковых систем (ГНСС). К тому же коммутатор выполняет ещё и функцию защиты входных цепей приёмника от электростатических разрядов.

Малошумящий усилитель (МШУ) в приёмном тракте выполняет первый этап усиления слабого принятого сигнала. От его линейности, шумовых и усилительных свойств зависят итоговые характеристики всего приёмника [1].

$$
-305-
$$




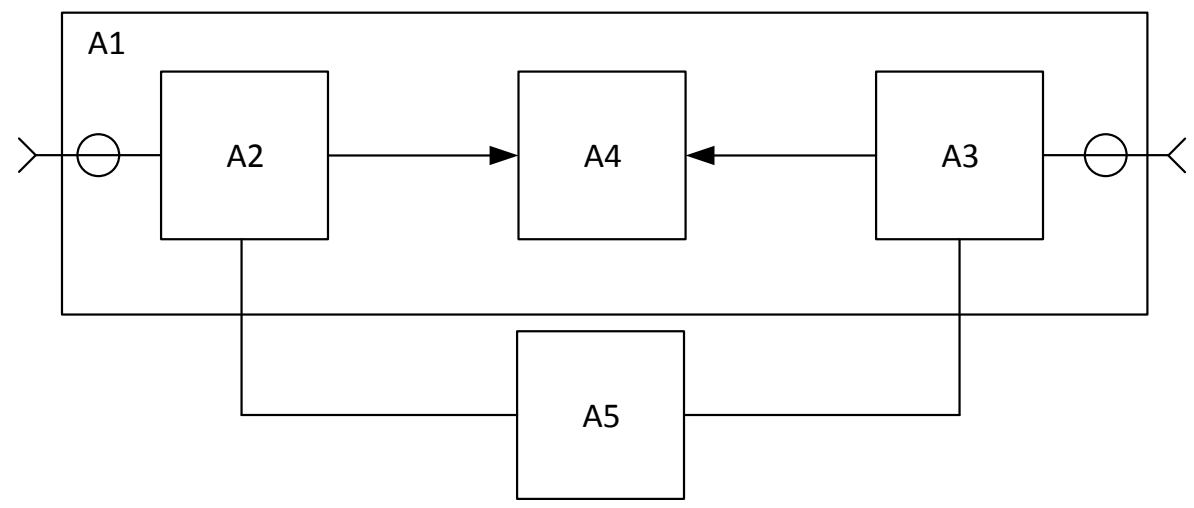

Рис. 1. Схема подключения объекта испытаний: A1 - зондовая станция Suss Micro Тес 200m; A2, A3 - СВЧ зонды Z040-K3N-GSG-150; A4 - объект испытаний; A5 - источник питания Agilent E 3646A

В данной статье приводятся результаты экспериментальных исследований электрических характеристик радиационностойких монолитно-интегральных схем (МИС) компонентов, а именно двухпозиционного коммутатора и МШУ, для применения в составе многоканальных ГНСС приёмников L-диапазона частот, разработанных и изготовленных на основе арсенидгаллиевой технологии собственного производства.

Ближайший зарубежный аналог GaAs MИC двухпозиционного коммутатора HMC190AMS8 производства компании Hittite Microwave (США) [2]. Ближайший отечественный аналог GaAs МИС двухпозиционного коммутатора - MP202 производства ЗАО «НПФ «Микран» (г. Томск) [3].

Ближайший зарубежный аналог GaAs МИС МШУ - HMC618LP3 производства компании Hittite Microwave (США) [4]. Ближайший отечественный аналог GaAs MИС МШУ - MP502 производства ЗАО «НПФ «Микран» (г. Томск) [5].

В дальнейшем на основании полученных результатов экспериментальных исследований планируется интеграция МШУ и двухпозиционного коммутатора на общий кристалл (Итерация 2).

Общим условием при проведении экспериментальных исследований являлось то, что измерение электрических характеристик кристаллов МИС проводили непосредственно на полупроводниковой пластине с помощью зондовой станции Suss Micro Tec 200m.

Схема измерения на основе зондовой станции приведена на рис. 1.

Двухканальный источник питания Agilent E 3646А служит для установки режима по постоянному току объектов испытаний.

\section{Исследования МИС МШУ}

Основными техническими характеристиками МИС МШУ TSR057 (ТУСУР) являются: коэффициент шума, коэффициент передачи, частотный диапазон, выходная мощность по сжатию коэффициента передачи на 1 дБ и ток потребления.

Измерение коэффициента шума МИС МШУ TSR057 (ТУСУР) проводили с помощью панорамного измерителя коэффициента шума Agilent N 8975А, позволяющего производить из-

$$
-306-
$$




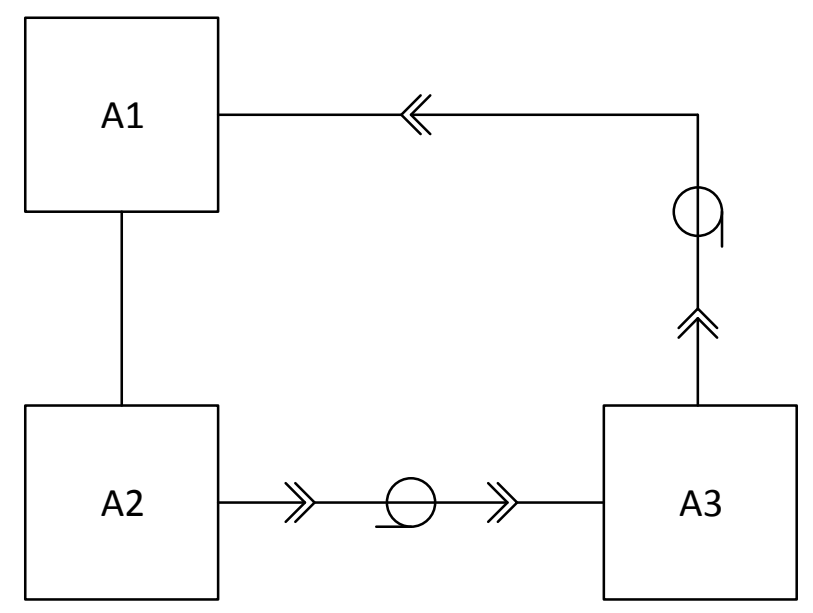

Рис. 2. Схема измерения коэффициента шума: A1 - измеритель коэффициента шума Agilent N 8975A; А2 - генератор шума Agilent N4002A; A3 - МИС МШУ

мерения в частотном диапазоне от 10,0 МГц до 26,5 ГГц. Погрешность измерения в диапазоне частот $\leq 3$ ГГц не превышает 0,05 дБ.

Схема измерения коэффициента шума с помощью Agilent N 8975А изображена на рис. 2.

Прибор Agilent N 8975А основан на методе Ү-фактора с коррекцией, являющемся основным методом для СВЧ-измерителей шума. Полное измерение коэффициента шума в данном случае состоит из двух шагов. Первый шаг называется калибровкой, которая производится без измеряемого устройства. На вход измерителя коэффициента шума подключают генератор шума (ГШ) с калиброванным значением избыточного коэффициента шума (ENR). В нашем случае использован ГШ фирмы Agilent N 4001A, основанный на эффекте возникновения шумового сигнала при лавинном пробое p-n-перехода диода, имеющий ENR от 4,5 до 6,0 дБ. При калибровке в прибор вводят таблицу ENR в точках частотного диапазона согласно маркировке ГШ. Для прибора Agilent N 8975А эта операция осуществляется автоматически, без участия оператора.

При проведении калибровки измеряют Ү-фактор, который определяется как отношение выходной мощности шума при включенном («On») и выключенном («Off») состоянии ГШ, а также шумовых температур, соответствующих данным режимам. Используя соотношение

$$
\mathrm{F}_{\mathrm{W}}=\mathrm{ENR} /(\mathrm{Y}-1)
$$

и

$$
\mathrm{ENR}=10 \lg \left[\left(\mathrm{T}_{1}-\mathrm{T}_{2}\right) / \mathrm{T}_{0}\right]
$$

где $\mathrm{T}_{1}$ - шумовая температура при включенном ГШ; $\mathrm{T}_{2}$ - шумовая температура при выключенном ГШ; $\mathrm{T}_{0}=290 \mathrm{~K}$ - опорная шумовая температура, определяют коэффициент шума прибора. В конце калибровки прибор сохраняет измеренные значения и приводит кривые изменения коэффициента шума к номинальному значению 0 дБ. После этого прибор готов к измерению электрических характеристик МИС МШУ TSR057 (ТУСУР).

$$
-307-
$$


Следующим после калибровки шагом является включение измеряемого устройства в разрыв между генератором шума и прибором и повторное измерение коэффициента шума методом У-фактора. При этом коэффициент шума измеряемого устройства определяется из условия

$$
\mathrm{F}_{1}=\mathrm{F}_{\mathrm{S}}-\left(\mathrm{F}_{2}-1\right) / \mathrm{K}_{\mathrm{P}}
$$

где $\mathrm{F}_{1}$ - коэффициент шума МИС МШУ; $\mathrm{F}_{\mathrm{S}}$ - коэффициент шума системы, состоящей из каскадного соединения МИС МШУ и прибора; $\mathrm{F}_{2}$ - коэффициент шума прибора; $\mathrm{K}_{\mathrm{P}}$ - коэффициент усиления МИС МШУ.

Представленный метод измерения позволяет с высокой точностью определять коэффициент шума в широком частотном диапазоне. Как уже отмечалось, в диапазоне до 3 ГГц погрешность измерения не превышает 0,05 дБ. При этом результаты измерения выводятся на экран прибора в виде численных значений и панорамных графиков.

В ходе проведения экспериментальных исследований были измерены опытные образцы МИС МШУ TSR057 (ТУСУР). Результаты измерения показали, что коэффициент шума МИС МШУ имеет значения в пределах от 2,0 до 2,5 дБ, что превосходит отечественный аналог (4,5 дБ), но уступает зарубежному (0,85-1,1 дБ). Это в первую очередь связано с технологией изготовления, в которую будут внесены соответствующие коррекции для достижения требуемого значения при следующей (второй) итерации.

Измерение коэффициента передачи и частотных характеристик МИС МШУ проводили с помощью векторного анализатора цепей Agilent N524 2AS, который относится к серии PNA$\mathrm{X}$. Отмеченный прибор позволяет измерять значительное число параметров (АЧХ, КСВ, ФЧХ, ГВ3, интермодуляционные искажения и т.д.) в частотном диапазоне от 10,0 МГц до 26,5 ГГц. Схема измерения АЧХ коэффициента передачи МИС МШУ представлена на рис. 3.

Перед проведением измерений, как и для измерения коэффициента шума, осуществяется калибровка прибора с устранением влияния адаптера (зондовой станции). Кроме того, методы калибровки предполагают коррекцию ошибок рассогласования на входе и выходе при измере-

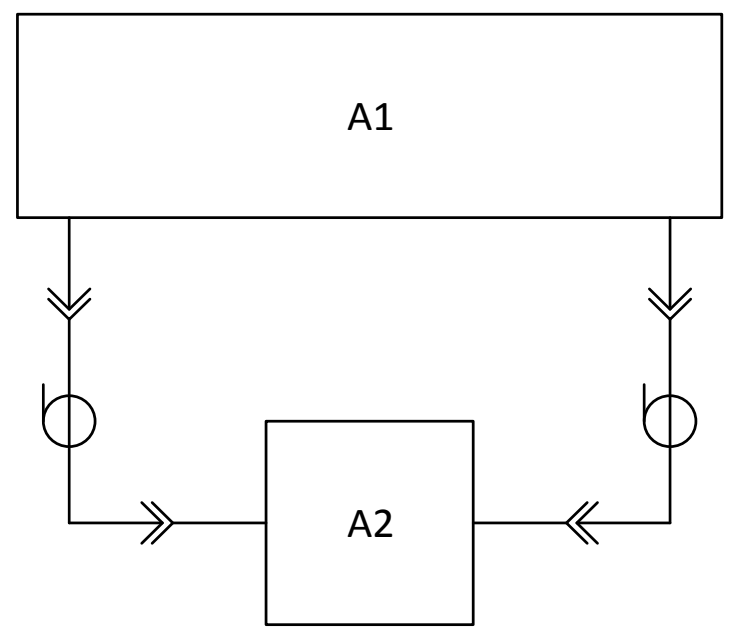

Рис. 3. Схема измерения АЧХ коэффициента передачи: A1 - векторный анализатор цепей Agilent N524 2AS; А2 - МИС МШУ 


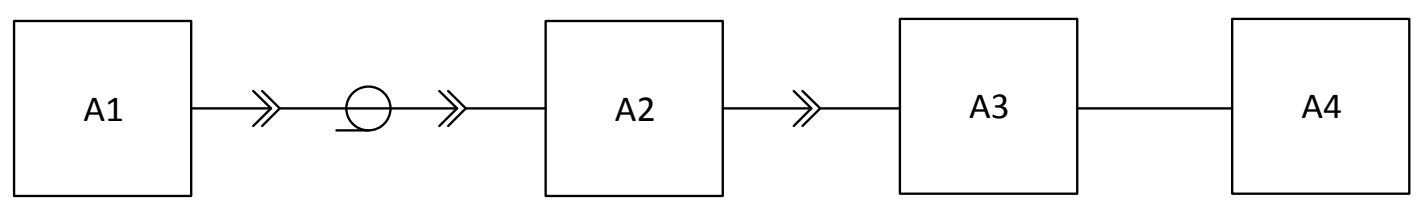

Рис. 4. Структурная схема измерения выходной мощности по критерию сжатия коэффициента передачи на 1 дБ: А1 - СВЧ-генератор; А2 - СВЧ-усилитель МАНW 020200; А3 - МИС МШУ; А4 - ваттметр Agilent N1911A

ниях параметров передачи, что значительно уменьшает неравномерность частотной характеристики и улучшает точность измерения, которая для коэффициента передачи $\leq 0,047$ дБ.

Было проведено измерение зависимостей АЧХ коэффициента передачи опытных образцов МИС МШУ TSR057 (ТУСУР). При этом величина коэффициента передачи изменялась в пределах 23-25 дБ, что существенно превосходит как отечественные (18-20 дБ), так и зарубежные (19 дБ) показатели. Частотный диапазон составил от 1,0 (нижняя граничная частота) до 2,5 ГГц (верхняя граничная частота) при значениях рассматриваемых аналогов: MР502 - от 1 до 4 ГГц, HMC618LP3 - от 1,2 до 1,7 ГГц.

Измерение выходной мощности по критерию сжатия коэффициента передачи на 1 дБ проводили в соответствии со структурной схемой измерения, изображенной на рис. 4.

В качестве СВЧ-генератора был использован СВЧ-выход анализатора цепей Agilent N524 $2 \mathrm{AS}$, который имеет при выходной мощности 0 дБмВт очень низкий уровень гармонических составляющих (минус 60 дБ). СВЧ-усилитель MAHW-020200 фирмы «Микран» служит для развязки и раскачки выходного сигнала генератора.

Выходную мощность измеряют СВЧ-ваттметром Agilent N1911A, работающим в частотном диапазоне от 50 МГц до 18 ГГц. Пределы измерения выходной мощности от $0,3 * 10^{-7}$ Вт до 0,1 Вт при относительной погрешности $\pm 5 \%$.

В начале измерения устанавливают относительно малый уровень входного воздействия (минус 40 дБмВт) и определяют малосигнальный коэффициент передачи по мощности:

$$
\kappa_{\mathrm{P}}(\text { ДБ })=10 \cdot \lg \left(\mathrm{P}_{\text {выХ }} / \mathrm{P}_{\text {ВХ }}\right) \text {. }
$$

По мере увеличения водной мощности за счёт нелинейных эффектов происходит уменьшение (сжатие) коэффициента передачи. Линейным считается режим, при котором сжатие коэффициента передачи не превышает 1 дБ. Для МИС МШУ TSR057 (ТУСУР) по отмеченной ме-

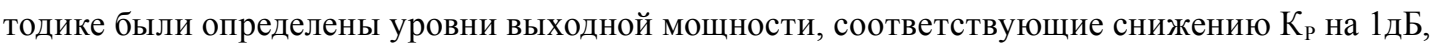
которые составили 14,0-14,8 дБм во всём частотном диапазоне. Данная величина соответствует показателю для отечественного (13,5 дБм) и зарубежного (14,5-10,0 дБм) аналогов.

Ток потребления, который фиксировался источником питания Agilent E 3646A, не превышал порог в 50 мА при соответствующем показателе MP502 в 80 мА и HMC618LP3 в 11865 мA.

\section{Исследования МИС двухпозиционного коммутатора}

Основными техническими характеристиками МИС двухпозиционного коммутатора TSR028 (ТУСУР) являются: вносимые потери открытого и закрытого канала, частотный диа-

$$
-309-
$$




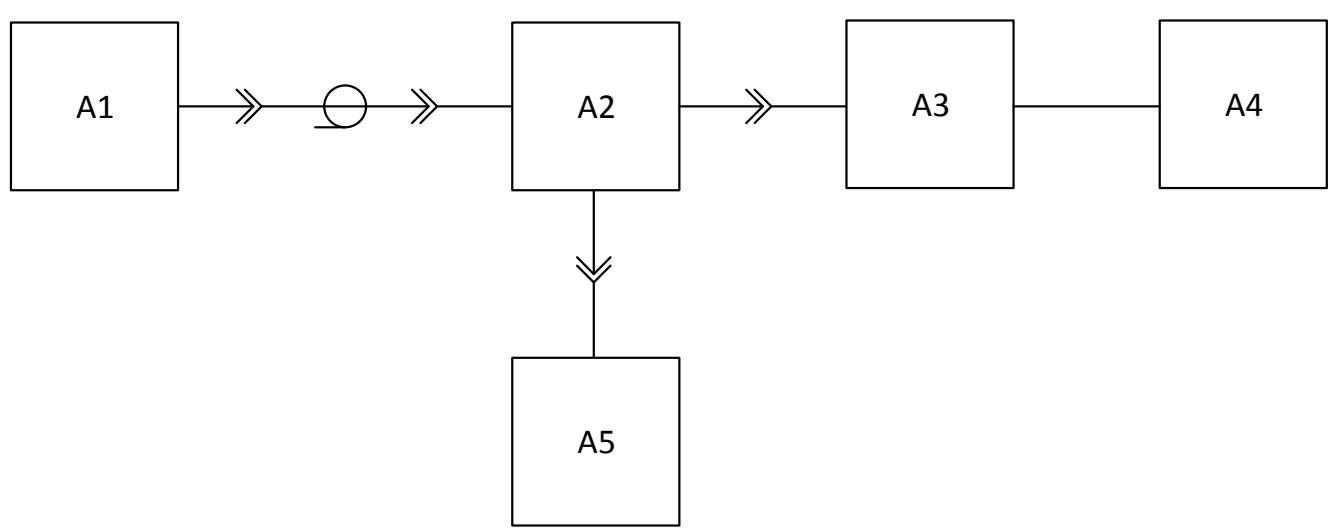

Рис. 5. Схема измерения вносимых потерь двухпозиционного коммутатора: A1 - СВЧ-генератор; A2 - двухпозиционный коммутатор; А3 - СВЧ-усилитель МАHW-020200; A4 - ваттметр Agilent N1911A; А5 - нагрузка 50 Ом

пазон, возвратные потери (коэффициент стоячей волны, КСВ), входная мощность по сжатию коэффициента передачи на 1 дБ, ток потребления.

Измерение основных характеристик МИС двухпозиционного коммутатора производится с помощью приборов, используемых и при измерении МИС МШУ. Отличительной особенностью признано то, что при проведении измерений передаточных характеристик открытого и закрытого канала необходимо подключение пятидесятиомной нагрузки в канал, не используемый для прохождения сигнала. Для её подключения применяют дополнительный СВЧ-зонд. Схема измерения вносимых потерь приведена на рис. 5.

Измерение вносимых потерь открытого канала производится при низком входном уровне СВЧ-генератора (минус 40 дБм). Чтобы не допустить дополнительных нелинейных искажений, СВЧ-усилитель из схемы измерения исключается. Потери определены в соответствии с выражением (4) и составили для всех образцов не более 0,5 дБ, что соответствует отечественному и зарубежному уровням.

Для закрытого канала вначале производится калибровка коэффициента передачи вместе с СВЧ-усилителем, а затем определяется суммарный коэффициент передачи, из которого исключается коэффициент усиления усилителя. В ходе проведенных измерений вносимые потери закрытого канала для всех образцов были более 40,5 дБ, что соответствует значению показателя для отечественного аналога (43-38 дБ) и существенно (в 1,8 раза) превышает для зарубежного (23 дБ).

Частотный диапазон составил от 0,1 (нижняя граничная частота) до 1,8 ГГц (верхняя граничная частота) при значениях рассматриваемых аналогов: МР202 - от 1 до 8 ГГц, HMC190AMS8 - от 0 до 3 ГГц.

Возвратные потери измеряли непосредственно с помощью векторного анализатора цепей Agilent N524 2AS по схеме, представленной на рис. 3. Как уже отмечалось, Agilent N524 2AS позволяет измерять значительное число параметров, в том числе возвратные потери (КСВ). Проведенные испытания показали, что возвратные потери для всех образцов изменялись в пределах минус 16 - минус 17 дБ, что на 8-3 дБ меньше, чем у зарубежной GaAs MИС (24-20 дБ), и на 6-7 дБ больше, чем у отечественной.

$$
-310-
$$


Входная мощность по сжатию коэффициента передачи на 1 дБ измерена для открытого канала по схеме, представленной на рис. 5, и составила не менее 16 дБм, что на 7-9 дБ ниже, чем у НMC190AMS8 (25-23 дБм) и МР202 (23 дБм).

Ток потребления, который фиксировался источником питания Agilent E 3646A, не превышал порога в 40 мкА при соответствующем показателе МР202 в 50 мкА.

\section{Заключение}

Таким образом, экспериментальные испытания показали, что разработаны схемотехнические и топологические решения экспериментальных образцов МИС двухпозиционного коммутатора и МШУ L-диапазона частот на основе собственной (НОЦ «Нанотехнологии» ТУСУР) GaAs pНЕМТ-технологии с параметрами, за исключением коэффициента шума, на уровне современных аналогов (табл. 1) для применения в составе многоканальных ГНСС приёмников.

Коэффициент шума разработанной GaAs МИС МШУ TSR057 (ТУСУР) уступает зарубежному аналогу. Возможные причины [6]:

- большая проводимость металлизации в сравнении со значением, принятым при проектировании. Как следствие, высокие потери во входной согласующей цепи, которые не могут быть компенсированы;

- несоответствие шумовой модели транзистора.

Таблица 1. Сводная таблица электрических характеристик разработанных МИС и их аналогов

\begin{tabular}{|c|c|c|c|c|c|c|c|}
\hline \multirow{9}{*}{$\underset{⿱ 乛}{\stackrel{\Xi}{\Xi}} \underset{\Xi}{\Xi}$} & \multirow{3}{*}{$\begin{array}{c}\text { Наименование параметра, } \\
\text { единица измерения }\end{array}$} & \multicolumn{6}{|c|}{ Значение } \\
\hline & & \multicolumn{2}{|c|}{ HMC618LP3 } & \multicolumn{2}{|c|}{ MP502 } & \multicolumn{2}{|c|}{ TSR057 } \\
\hline & & Мин. & Макс. & Мин. & Макс. & Мин. & Макс. \\
\hline & Граница рабочей полосы частот, ГГц & 1,2 & 1,7 & 1 & 4 & 1,0 & 2,5 \\
\hline & Коэффициент усиления, дБ & 19 & - & 18 & 20 & 23 & 25 \\
\hline & Коэффициент шума, дБ & 0,85 & 1,1 & - & 4,5 & 2,0 & 2,5 \\
\hline & $\begin{array}{l}\text { Выходная мощность при сжатии КП на } \\
1 \text { дБ, дБм }\end{array}$ & 10,0 & 14,5 & 13,5 & - & 14,0 & 14,8 \\
\hline & Напряжение питания, В & 3 & 5 & - & 5 & - & 5 \\
\hline & Ток потребления, мА & 65 & 118 & - & 80 & 45 & 50 \\
\hline \multirow{8}{*}{ 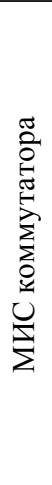 } & & \multicolumn{2}{|c|}{ HMC190AMS8 } & \multicolumn{2}{|c|}{ MP202 } & \multicolumn{2}{|c|}{ TSR028 } \\
\hline & Граница рабочей полосы частот, ГГц & 0 & 3 & 1 & 8 & 0,1 & 1,8 \\
\hline & $\begin{array}{l}\text { Вносимые потери открытого канала, } \\
\text { дБ }\end{array}$ & - & 0,6 & 0,3 & 0,6 & - & 0,5 \\
\hline & Вносимые потери закрытого канала, дБ & 23 & - & 38 & 43 & 40,5 & - \\
\hline & $\begin{array}{l}\text { Входная мощность при сжатии КП на } \\
1 \text { дБ, дБм }\end{array}$ & 23 & 25 & 23 & - & 16 & - \\
\hline & Возвратные потери, дБ & 20 & 24 & 10 & - & 16 & 17 \\
\hline & Управляющее напряжение, В & 0 & $3 \ldots 8$ & 0 & $3,3 \ldots 5$ & 0 & 5 \\
\hline & Ток потребления, мкА & - & - & - & 50 & - & 40 \\
\hline
\end{tabular}


Для устранения указанного недостатка в дальнейшем планируется:

- провести измерения тестовых пассивных элементов (отрезок микрополосковой линии, спиральная катушка индуктивности) и уточнить значение удельной проводимости металлизации. Результаты использовать в процессе интеграции малошумящего усилителя и двухпозиционного коммутатора на общий кристалл (Итерация 2);

- провести измерения параметров тестовых транзисторов в различных режимах по постоянному току. Определить режим работы, соответствующий минимальному коэффициенту шума. Уточнить шумовую модель транзистора. Результаты использовать в процессе интеграции малошумящего усилителя и двухпозиционного коммутатора на общий кристалл (Итерация 2).

Работа выполнена в "НОЦ «Нанотехнологии» ТУСУР в рамках реализации постановления Правительства РФ от 09.04.2010 № 218 и договора между ОАО «ИСС» И Минобрнауки РФ от 12.02.2013 № 02.G 25.31.0042 [7].

\section{Список литературы}

[1] Дмитриев В.Д., Терешков В.В., Саяпин В.Ю. // Доклады ТУСУР. 2014. № 3(33). С. 16-21.

[2] HMC190AMS8/190AMS8E - GaAs MMIC SPDT Switch DC-3 Ghz [Электронный pecypc]. - Режим доступа: http://www.radiant.su/other/hittite/pdf/hmc190ams8.pdf, свободный (дата обращения: 10.06.2013).

[3] GaAs МИС двухпозиционного СВЧ-коммутатора MP202 [Электронный ресурс]. - Режим доступа: http://www.micran.ru/productions/MIS/switches/MP202, свободный (дата обращения: 10.06.2013).

[4] HMC618LP3/618LP3E - GaAs SMT pHEMT Low Noise Amplifier, $1.2-2.2 \mathrm{GHz}$ [Электронный ресурс]. - Режим доступа: http://www.radiant.su/other/hittite/pdf/hmc618lp3.pdf, свободный (дата обращения: 10.06.2013).

[5] GaAs МИС широкополосного усилителя 1-4 ГГц MP502 [Электронный ресурс]. - Режим доступа: http://www.micran.ru/productions/MIS/amplifiers/MP502, свободный (дата обращения: 10.06.2013).

[6] Зыков Д.Д., Матвеев В.В., Нечаев К.А., Карабан В.М. // Доклады ТУСУР. 2013. № 4(30). C. $173-176$.

[7] Куприи В.Ю., Шарыгин Г.С., Школьный В.Н. // Доклады ТУСУР. 2014. № 3(33). С. 22-26. 